\title{
Detección Temprana Mediante PCR de Patógenos Periodontales en Relación a Implantes Oseointegrados
}

\author{
Early Detection of Periodontal Pathogens by \\ PCR in Relation to Osseointegrated Implants
}

\author{
Iván Godoy*; Juan Pablo Parrochia**; Mauricio Bittner ${ }^{* \star *}$ \& José Valdivia***
}

GODOY, I.; PARROCHIA, J. P.; BITTNER, M. \& VALDIVIA, J. Detección temprana mediante PCR de patógenos periodontales en relación a implantes oseointegrados. Int. J. Odontostomat., 8(3):405-412, 2014.

RESUMEN: El objetivo de este trabajo fue valuar la presencia temprana en sitios dentarios e implantarios de cuatro bacterias periodontopatógenas ( $A$. actinomycetemcomitans, $P$. gingivalis, $T$. forsythensis y $T$. denticola) luego de dos semanas de la cirugía de segunda fase, además se propone comparar la presencia de estas 4 bacterias en sitios subgingivales dentarios e implantarios. Se estudiaron mediante reacción en cadena de polimerasa muestras de placa subgingival de implantes y de dientes vecinos a ellos, dos semanas luego de la cirugía de segunda fase. Dieciséis implantes y trece dientes en diez pacientes fueron seleccionados. Luego de dos semanas se encontró presencia de bacterias periodontopatógenas en sitios tanto periodontales como peri-implantarios, no se encontró relación entre diente e implante para $P$. gingivalis, T. forsythensis y $T$. denticola. Se encontró una relación significativa para $A$. actinomycetemcomitans $(\mathrm{P}<0,005)$. La bacteria detectada con mayor frecuencia fue $P$. gingivalis y la menos encontrada fue $T$. denticola. La dependencia para $A$. actinomycetemcomitans estuvo relacionada a pacientes con historia de periodontitis. Dentro de los límites de este estudio, los resultados muestran la presencia temprana de los cuatro patógenos periodontales alrededor de los sitios implantarios, y una relación estadísticamente significativa $(P<0,005)$ entre implante y diente para $A$. actinomycetemcomitans.

PALABRAS CLAVE: patógenos periodontales, PCR, implantes oseointegrados.

\section{INTRODUCCIÓN}

Se considera que la cavidad oral del ser humano es el nicho ecológico con mayor biodiversidad conocido hasta ahora. En el año 2001 se estimaba que existían 500 especies en la cavidad oral, hoy se calcula que serían por lo menos 700 (Perea, 2004). Aun así se considera que se conoce realmente solo un 50 $60 \%$ de las bacterias que se encuentran en la cavidad oral (Artimage, 2010).

Algunos estudios han demostrado la presencia de elevados niveles de patógenos periodontales a nivel subgingival alrededor de implantes dentales afectados por pérdida ósea y formación de sacos periimplantarios (Hultin et al., 2002; Botero et al., 2005), por lo que se ha propuesto que los dientes adyacentes, periodontalmente comprometidos, podrían servir como reservorios microbianos, desde donde se inicia la colonización de la superficie del implante. No obstante lo anterior, surge la interrogante de si este mismo efecto de reservorio microbiano ocurre con dientes de pacientes sin antecedentes de enfermedad periodontal, o en aquellos casos en los cuales la enfermedad ya fue tratada, considerándose el paciente en normalidad.

El $6^{\circ}$ Workshop Europeo de Periodoncia realizado el año 2008 confirma que la enfermedad periimplantaria es de naturaleza infecciosa. Describe, además, la mucositis periimplantaria, como una lesión inflamatoria que reside en la mucosa que rodea el implante, mientras que la periimplantitis afecta además el hueso de soporte (Lindhe et al., 2008).

* Residente Programa de Especialización en Implantología, Universidad Andrés Bello, Santiago, Chile.

** Profesor Asociado Programa de Especialización en Implantología, Universidad Andrés Bello, Santiago, Chile.

** Profesor Asistente Laboratorio de Microbiología y Biotecnología Oral, Universidad Andrés Bello, Santiago, Chile.

**** Profesor Titular, Universidad Autónoma de Chile, Santiago, Chile. 
La prevalencia de peri-implantitis varía entre 1 y 19\% (Roos-Jansåker et al., 2003). Los datos sugieren que pacientes con una historia de periodontitis parecen ser más susceptibles a desarrollar infecciones periimplantarias (Van der Weijden et al., 2005), reportándose un $8 \%$ de fracaso en este tipo de pacientes vs. un $3 \%$ para los pacientes no susceptibles luego de 5 años (Hardt et al., 2002). Se ha demostrado que la colonización de los surcos periimplantarios con bacterias asociadas con periodontitis, ocurre dentro de las dos primeras semanas (Quirynen et al., 2006). El patrón de colonización temprana podría contribuir y ser explicatorio para el desarrollo de las lesiones periimplantarias (Fürst et al., 2007).

Se ha observado la presencia de $P$. gingivalis, $P$. intermedia, A. actinomycetemcomitans, C. rectus y especies Capnocytophaga en sitios que parecen sanos, sin embargo la presencia de estos patógenos periodontales y la ausencia de signos clínicos, podría ser solo una situación temporal que podría evolucionar a una peri-implantitis (Agerbaek et al., 2006).

Existe insuficientes datos en relación a la colonización temprana y sobre la formación de biofilm alrededor de los implantes, y ninguna información sobre el impacto de las bacterias presentes en los dientes adyacentes. Si bien no hay consecuencias de la presencia de bacterias por los primeros seis meses, esa presencia temprana podría tener consecuencias futuras (Fürst et al.).

Hoy en día, la oseointegración es un hecho que ya no se cuestiona, por lo que la implantología fija su atención en los factores que pudiesen afectar esta oseointegración ya lograda. El poder detectar cualquier situación de manera temprana, y modificar los protocolos de diagnóstico y mantención de estos implantes se hace de suma importancia en los tiempos actuales.

Esta investigación pretende evaluar si existe presencia temprana de patógenos periodontales, en los dientes de pacientes parcialmente desdentados, sometidos a tratamiento de implantes dentales, y relacionarla con los patógenos encontrados alrededor de los implantes previamente instalados.

\section{MATERIAL Y MÉTODO}

Para este estudio se seleccionaron 10 pacientes, 8 mujeres y 2 hombres, atendidos en el Postgrado de Implantología de la Universidad Andrés Bello, Santiago de Chile. Estos pacientes recibieron implantes endóseos, Brånemark System (Nobel Biocare ${ }^{\circledR}$, Gotemburgo, Suecia) de dos fases quirúrgicas, para tratar regiones parcialmente edéntulas. Dos semanas después de realizada la cirugía de conexión de pilares de cicatrización, se tomaron las muestras.

Las edades de los pacientes fluctuaron entre los 24 y 73 años de edad. Todos los sujetos se encontraban en buen estado de salud general, sin focos activos de enfermedad periodontal o caries. Se verificó que los pacientes no hayan tomado antibióticos por lo menos por 3 meses, antes de tomar las muestras.

Se seleccionó el pilar de mayor largo dentro de los que el paciente pudiese poseer como primer objeto de muestra, y luego se seleccionó el diente más próximo a este como segundo objeto de muestreo y finalmente se seleccionó como tercer objeto de muestra el diente o implante que estuviera más cercano al primer implante. Los sitios de toma de muestra fueron seleccionados en base a las radiografías prequirúrgicas de conexión de cada paciente, para así no alterar el biofilm presente, si se hiciese el sondaje de los surcos.

Previo aislamiento relativo de los dientes, se realizó mediante instrumentación manual, el destartraje supragingival de los sitios seleccionados. Luego de secar el área con una gasa estéril, se insertaron dos conos de papel estéril ISO \#40 (Denstply) a la vez, buscando llegar a la zona más profunda del surco periodontal o periimplantario elegido, ingresándolos en un movimiento único y paralelo al eje del diente o del implante, y dejándolos dentro del surco por un tiempo de 15 segundos. Luego de retirar los conos de papel, se depositaron en un tubo Eppendorf debidamente identificado, para luego ser llevado al laboratorio de microbiología de la misma Universidad Andrés Bello, donde fueron procesadas las muestras.

Análisis Estadístico. Los datos demográficos, clínicos y microbiológicos fueron analizados mediante el software estadístico SPSS 16.0 para Windows (SPSS Inc., Wacker Drive, Chicago, USA). Los datos descriptivos fueron expresados en porcentajes, promedios y desviación estándar. Diferencias en la frecuencia de detección de microorganismos fueron analizadas mediante las pruebas de chi cuadrado y exacta de Fisher. La significancia estadística se estableció al 0,05 ( $p<$ valor) con intervalos de confianza al 95\%. 


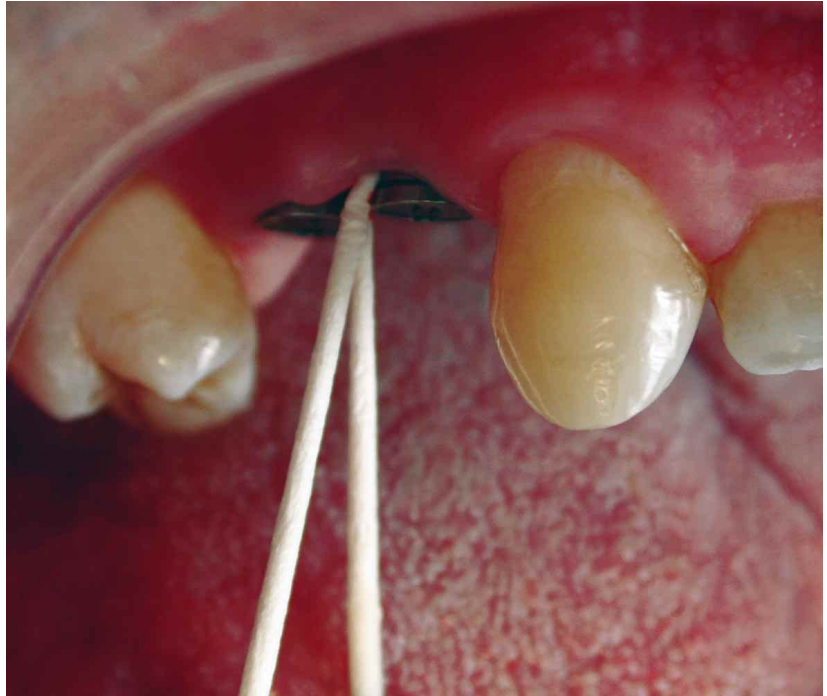

Fig. 1. Conos de papel en surco periimplantario.

\section{RESULTADOS}

De los 10 pacientes, con uno o más implantes, que fueron incluidos en este trabajo, se seleccionaron para el estudio microbiológico 16 implantes y 14 dientes. Tanto dientes como implantes se encontraban sanos al examen clínico y radiográfico. En la Tabla I, se detallan las características demográficas y clínicas de los pacientes.
En la Tabla II se presenta la frecuencia de detección de los diferentes microorganismos detectados a través de PCR, registrado durante la toma de muestra luego de dos semanas después de la cirugía de conexión de los implantes.

Al observar la Tabla II, se reconoce una mayor frecuencia de detección de patógenos periodontales en implantes que en dientes naturales. Sin embargo esta diferencia no se presenta como estadísticamente significativa $(p=0,533)$. T. forsythia, $P$. gingivalis y $A$. actinomycetemcomitans, se encontraron con mayor frecuencia alrededor de implantes que en dientes; $T$. denticola se encontró con la misma baja frecuencia en ambos. La especie bacteriana encontrada con mayor frecuencia de detección fue $P$. gingivalis, tanto en dientes como en implantes, mientras que la encontrada en menor cantidad fue $T$. denticola, de igual modo en dientes e implantes.

Se utilizó la prueba de chi cuadrado para comprobar las diferencias en la frecuencia de detección de periodontopatógenos en implantes y dientes dentro de un paciente. Para el análisis estadístico se escogió el pilar de cicatrización de mayor longitud dentro de cada paciente y el diente más próximo a este.

Al comparar las frecuencias para cada especie bacteriana, sólo en el caso de A. actinomycetemcomitans,

Tabla I. Epidemiología descriptiva de los pacientes analizados en el estudio.

\begin{tabular}{llcc}
\hline & & Frecuencia & Porcentaje \\
\hline Total de Pacientes & & 10 & $100 \%$ \\
Sexo & Masculino & 2 & $20 \%$ \\
Edad & Femenino & 8 & $0 \%$ \\
& Mínima & 24 & Prom: 56,75 \\
Fumador & Máxima & 69 & DE $=14,26$ \\
& Si & 1 & $10 \%$ \\
Antecedentes de & No & 9 & $90 \%$ \\
Enfermedad Periodontal & Si & 3 & $30 \%$ \\
& No & 7 & $70 \%$ \\
\hline
\end{tabular}

Tabla II. Frecuencia de detección de periodontopatógenos en implantes y dientes analizados en el estudio.

\begin{tabular}{lcc}
\hline & Implantes $(\mathbf{n = 1 6 )}$ & Dientes $(\mathbf{n = 1 3})$ \\
\hline T. dentícola & $1(6,25 \%)$ & $1(7,69 \%)$ \\
T. forrsythia & $7(43,75 \%)$ & $3(23,07 \%)$ \\
$\boldsymbol{P}$. gingivalis & $9(56,25 \%)$ & $7(53,84 \%)$ \\
A. actinomycetemcomitans & $3(18,75 \%)$ & $2(15,38 \%)$ \\
\hline
\end{tabular}


se observó una dependencia estadísticamente significativa entre dientes e implantes ( $c 2=10,00, \mathrm{gl}=1$, $p=0,002$; prueba exacta de Fisher $p=0,022$ ). Se rechaza la hipótesis nula respecto a independencia, y se establece que existe asociación de dependencia entre $A$. actinomycetemcomitans detectado en implantes, respecto al detectado en dientes adyacentes de un mismo paciente, en esta muestra de este estudio. El resto de las especies bacterianas mostró independencia entre la frecuencia de detección de implantes respecto de dientes $(p>0,05)$.

En vista de las diferencias entre las características de los individuos, decidimos probar como se modifican los valores de la prueba de independencia al homogeneizar la muestra sobre la cual se realiza el análisis. Primero, al analizar los datos respecto a la calidad de fumador del paciente, vemos que sólo uno de ellos declara ese hábito. Al excluir a este paciente del análisis, vemos que igualmente se observa una asociación de dependencia sólo en el caso del $A$. actinomycetemcomitans (2-sided exact $\mathrm{P}=0,0278$ ).

Nuevamente al analizar las características de la muestra de los pacientes incluidos en el estudio, vemos que la mayor proporción de ellos son mujeres, con sólo 2 varones. Al excluir a estos últimos y probar la posibilidad de alguna asociación relacionada al sexo femenino, observamos que se mantiene la tendencia a mostrar dependencia entre $A$. actinomycetem comitans detectado en implantes y en dientes, no obstante la fuerza de la asociación disminuye levemente (2-sided exact $\mathrm{P}=0,0357$ ).

Finalmente, al excluir el análisis aquellos pacientes con antecedentes de enfermedad periodontal (3), vemos que se pierde la relación de dependencia con A. actinomycetemcomitans (el resto de las especies bacterianas sigue mostrando independencia).

\section{DISCUSIÓN}

Se observa la presencia de los cuatro periodontopatógenos investigados, después de dos semanas de la cirugía de conexión. Estos hallazgos concuerdan con la mayoría de las publicaciones consultadas, siendo el único autor que discrepa al respecto Heuer et al. (2007). Estos autores, analizaron mediante PCR la presencia de Haemophilus actinomycetemcomitans, sinónimo de Aggregatibacter actinomycetemcomitans (Norskov-Lauritsen \& Kiliany,
2006), y P. gingivalis, dos semanas después de la conexión, en 14 implantes colocados en 10 pacientes, obteniendo las muestras mediante conos de papel. El estudio no encontró presencia de estas bacterias en ninguna de las muestras. La explicación de esto, según los autores, es que se requiere de un biofilm ya maduro y de la proliferación bacteriana subgingival, situación que no se dio debido posiblemente a la formación de una barrera periimplantaria gracias a fibras colágenas circulares y hemidesmosomas, también al estado saludable del periodonto de los dientes adyacentes. En relación a lo mismo, Fürst et al., realizando un estudio mediante PCR en fluido peri-implantario de 17 implantes evaluados, observaron colonización bacteriana a partir de los 30 minutos luego de colocar los implantes.

De Boever \& De Boever (2006), en un estudio realizado sobre 68 implantes colocados en 22 pacientes con antecedentes de periodontitis agresiva, donde las muestras fueron obtenidas mediante conos de papel y procesadas mediante PCR múltiple, observaron la presencia temprana de patógenos periodontales ya desde la primera semana.

Quirynen et al. en su estudio sobre 42 pacientes parcialmente desdentados, también observaron presencia de patógenos periodontales a partir de la primera semana, incluso en pacientes en tratamiento con colutorio de clorhexidina, es más, luego de la segunda semana no se observan mayores cambios en cuanto al número de especies que componen la microbiota subgingival. Las muestras fueron tomadas con conos de papel y analizadas mediante cultivo, PCR e hibridización DNA-DNA. Según los mismos autores, a pesar de no ser el único modo de transmisión, los dientes actuarían como reservorios para la formación del biofilm subgingival alrededor de los implantes.

Con respecto a si existe diferencia entre implantes dentales y dientes naturales en relación a los periodontopatógenos más importantes ( $T$. denticola, $T$. forsythia, P. gingivalis y A. actinomycetemcomitans), los resultados obtenidos no logran establecer una dependencia de manera significativa entre la flora presente alrededor de los implantes y los dientes cercanos, salvo para el caso de $A$. actinomycetemcomitans, donde la relación si se estableció como estadísticamente significativa. En relación a esto último, los resultados observados en la literatura son diversos, si bien a nuestro parecer, se observa una mayor propensión a desacreditar la relación entre flora 
de dientes e implantes en la segunda mitad de esta década, la cual se observaba de forma bastante más clara y directa a fines de los años 90 y principios del año 2000.

El concepto de que las lesiones periimplantarias están relacionadas con patógenos específicos asociados con la periodontitis está basado en observaciones de diversos estudios que envuelven a unos pocos sujetos (Botero et al.; Leonhardt et al., 1999). Estos autores asumen que los patrones de colonización bacteriana en dientes existentes, predicen la colonización de sitios implantarios. Si las bacterias asociadas con periodontitis están también asociadas con infecciones periimplantarias no ha sido, explícitamente, demostrado. Otros estudios apoyan el concepto de transmisión o "translocación" de bacterias desde dientes a implantes (Sumida et al., 2002; Leonhardt et al., 2003). Es de ahí que bacterias asociadas con periodontitis podrían causar periimplantitis en pacientes que tienen tanto dientes como implantes. Se ha mostrado que sujetos que previamente eran portadores de patógenos periodontales puedan haber transmitido estos desde sacos residuales a sitios de implantes. Incluso luego de 10 años, los sujetos continuaban siendo portadores de estos patógenos (Leonhardt et al., 2002). Para autores como Leonhardt et al. (1999) o Renvert et al. (2007) la microflora alrededor de implantes y dientes es similar.

La colonización microbiana de implantes dentales podría llevar a una infección de los tejidos periimplantarios, con resultado depérdida ósea y eventualmente fallas de los implantes. También se ha demostrado que el tener altos niveles de estas bacterias puede asociarse con un riesgo incrementado de periimplantitis (Rutar et al., 2001). Consecuentemente, la ausencia o el pobre tratamiento de la enfermedad periodontal aumentarían el riesgo de infección periimplantaria (Karoussis et al., 2003). Se han mostrado distintas diferencias, cuantitativas y cualitativas, asociadas con implantes exitosos y fallidos (Leonhardt et al., 2002).

Fumar también parece ser un factor de riesgo para el tratamiento exitoso con implantes dentales (Leonhardt et al., 2002; Karoussis et al.). Este mal hábito ha sido asociado tanto con periodontitis como con complicaciones implantarías (Leonhardt et al., 2002; Bergström, 2003; Gruica et al., 2004). El fenómeno es que dentro de los fumadores, más pacientes mantienen cultivos positivos para patógenos periodontales luego de la terapia puede contribuir a los resultados desfavorables frecuentemente observados en pacientes fumadores con periodontitis (Van der Velden et al., 2003). Otros factores, como el polimorfismo para el gen de la interleuquina-1 o la periodontitis crónica, en combinación con fumar parecen conferir un alto riesgo combinado, para la pérdida de hueso periimplantario (Feloutzis et al., 2003).

El riesgo relativo para la progresión de la enfermedad no depende solo de la presencia de los periodontopatógenos sino también de su concentración y mantención (De Boever \& De Boever). Kocar et al. (2010), establecen que la presencia de patógenos periodontales en surcos periimplantarios no se correlaciona estadísticamente con su frecuencia alrededor de los dientes. De igual manera, Fürst et al., observaron patrones de colonización distintos al comparar dientes e implantes, sobre todo en los casos de $A$. actinomycetemcomitans y $P$. gingivalis. Según la autora, esto se explicaría debido a que existirían distintos medios por los cuales las bacterias colonizan los implantes más allá de la transmisión de diente a implante, medios que aún deben ser estudiados.

En nuestro estudio, llama la atención el que no se haya obtenido patógeno alguno, en los sitios dentarios de dos pacientes, y sin embargo, las muestras de los implantes de estos pacientes hayan dado positivo para $T$. forsythia en ambos casos, y $P$. gingivalis en uno de ellos. Si bien, en general se observó mayor presencia de patógenos periodontales en implantes que en dientes, esta diferencia no es significativa.

Por su parte, Nowzari et al. (2008) encontraron mayor frecuencia en la detección y en los niveles de periodontopatógenos alrededor de los dientes que en los implantes. P. gingivalis, $T$. forsythia y especies de Fusobacterium fueron las especies más representativas, mientras que $P$. intermedia estaba elevada alrededor de los implantes. En ningún caso la diferencia fue estadísticamente significativa.

En relación a la dependencia encontrada con respecto a $A$. actinomycetemcomitans, podemos decir, que se observó presencia de este patógeno, tanto en dientes como en implantes en dos de los pacientes evaluados, siendo el único patógeno encontrado en todos los sitios tanto dentarios como implantarios de estos pacientes. Otro dato importante, es que ambos pacientes tenían antecedentes de periodontitis, ya tratada con anterioridad. A pesar de lo anterior, el porcentaje de implantes encontrados con $A$. actinomycetemcomitans (18\%) es similar al encontra- 
do por Kocar et al. (15\%). Según Mombelli \& Décaillet (2011), A. actinomycetemcomitans se asocia de manera poco frecuente con la periimplantitis. La literatura sugiere que se deben considerar otros actores, más allá de los clásicos periodontopatógenos, de la enfermedad periimplantaria. Es así como estudios longitudinales han mostrado como $S$. aureus colonizaría tempranamente los implantes luego de ser colocados (Fürst et al.), y podría persistir por un largo periodo de tiempo (Salvi et al., 2008).

Se ha demostrado que rápidamente luego de la instalación de implantes de titanio, se establece una microbiota subgingival dominada por Peptostreptococcus micros, Fusobacterium nucleatum y Prevotella intermedia (van Winkelhoff et al., 2000). La energía libre superficial del titanio facilitaría la formación de biofilms bacterianos (Teughels et al., 2006). La presencia de bacterias asociadas con periodontitis como P. gingivalis, Prevotella intermedia, A. actinomycetemcomitans, Campylobacter rectus y especies Capnocytophaga ha sido reportada en sitios de implantes que parecen estar sanos (Leonhardt et al., 2002). La complejidad de los patrones de infección en implantes puede ser ilustrada de mejor forma por el hecho de que aunque se identificaron en niveles bajos $P$. gingivalis y $A$. actinomycetemcomitans, Buchmann et al. (2003) reportaron que la colonización microbiana de implantes era compatible con la salud de los tejidos periimplantarios.

El antecedente de periodontitis o la presencia actual de la enfermedad en los dientes remanentes tendría un impacto significativo en la microbiota periimplantaria (Gerber et al., 2006). Estos pacientes tendrían un mayor riesgo de infección cruzada con bacterias desde los sitios periodontales a los periimplantarios.

Se debe tener en cuenta que si bien la tecnología de PCR tiene un gran rol como herramienta adjunta de diagnóstico, las técnicas de cultivo siguen manteniendo algunas capacidades inherentes, como la posibilidad de detectar al mismo tiempo múltiples especies bacterianas, detectar bacterias por casualidad o determinar la resistencia antibiótica, lo que las mantiene como el estándar de referencia en microbiología periodontal (Quirynen et al.). Adicionalmente, no se puede dejar de considerar que las diferencias en los resultados obtenidos entre distintos investigadores puedan explicarse en parte por los distintitos tipos de sistemas de implantes utilizados por los investigadores (Fürst et al.).
Los resultados obtenidos en este estudio, al igual que en la mayoría de los estudios revisados, demuestran la presencia de bacterias en el fluido gingival y no en el biofilm posiblemente adherido a la superficie de los implantes. Al respecto, Gerber et al., proponen que la mejor forma de realizar este tipo de investigaciones sería mediante análisis del fluido crevicular con conos de papel y además, del biofilm, obtenido mediante cureta. Según los mismos autores, la mayoría de los estudios, muestran similitudes entre dientes e implantes cuando el método de toma de muestra es mediante el análisis de fluido crevicular, caso que no es el del presente estudio, ni el de Fürst et al. o de Kocar et al. En el mismo estudio se observa mayor proporción de periodontopatógenos en el líquido crevicular con respecto al biofilm, con la excepción de $P$. gingivalis que se encontró en mayor proporción en las muestras obtenidas con cureta. La mayoría de los estudios en relación a biofilm en implantes se realiza mediante muestras con conos de papel debido a la dificultad de obtener muestras de biofilm desde la pared del implante, mediante el uso de curetas. Se asume que cualquiera sea la muestra obtenida, esta presenta un grado de contaminación, ya sea con biofilm o fluido crevicular, además de bacterias contenidas en el epitelio de unión, que sería insignificante.

Nuestro estudio no logró establecer una relación entre la presencia de patógenos periodontales en los surcos periodontales y los periimplantarios del mismo paciente, con excepción de $A$. actinomycetemcomitans. En este aspecto coincidimos con lo expresado por Agerbaek et al., en el sentido que se requiere de más estudios para comprender de mejor manera el rol de la infección oral en los sujetos parcialmente dentados con implantes.

Si bien la mayoría de los patógenos periodontales están asociados con la peri-implantitis, su rol etiológico exacto, es aún desconocido. Es necesario establecer en futuras investigaciones, la participación de otras bacterias y la biología de los tejidos orales en contacto con los implantes de titanio (Kocar et al.).

\section{CONCLUSIONES}

Se aprecia colonización temprana por parte de los cuatro periodontopatógenos evaluados en los sitios periimplantarios, pero no se logró establecer dependencia entre los periodontopatógenos encontrados 
en los dientes vecinos, y las especies bacterianas ubicadas en los surcos periimplantarios, con excepción del A. actinomycetemcomitans. La mera presencia de es- tas bacterias encontradas, no permite relacionarlas con una flora patógena para los implantes, ni menos, permite establecer un pronóstico a futuro de los mismos.

GODOY, I.; PARROCHIA, J. P.; BITTNER, M. \& VALDIVIA, J. Early detection of periodontal pathogens by PCR in relation to osseointegrated implants. Int. J. Odontostomat., 8(3):405-412, 2014.

ABSTRACT: The aim of this study was to assess the early colonization of four periodontopathogenic bacteria $(A$. actinomycetemcomitans, $P$. gingivalis, $T$. forsythensis and $T$. denticola) on titanium implants immediately after two weeks following second stage surgery; to compare the presence of these four periodontopathogenic bacteria at subgingival implant and adjacent tooth sites. Subgingival plaque samples from implant and neighboring teeth were studied by PCR after two weeks following second stage surgery. Sixteen implants and thirteen teeth, in ten patients were selected. At two weeks, pathogenic bacteria presence was found in both peri-implant and periodontal sites, there was no relation found between tooth and implant for $P$. gingivalis, T. forsythensis and T. denticola. A significant relation was found for $A$. actinomycetemcomitans $(\mathrm{P}<0.005)$. The more frequently detected bacteria were $P$. gingivalis, and the less was $T$. denticola. The dependence for $A$. actinomycetemcomitans was related to patients with a history of periodontitis. Within the limits of this study, the findings showed the early presence of the four periodontopathogenic bacteria around implant sites and a statistically significant $(\mathrm{P}<0.005)$ relation between implants and teeth sites for $A$. actinomycetemcomitans.

KEY WORDS: periodontal pathogens, PCR, osseointegrated Implants.

\section{REFERENCIAS BIBLIOGRÁFICAS}

Agerbaek, M. R.; Lang, N. P. \& Persson, G. R. Comparisons of bacterial patterns present at implant and tooth sites in subjects on supportive periodontal therapy. I. Impact of clinical variables, gender and smoking. Clin. Oral Implants Res., 17(1):18-24, 2006.

Armitage, G. C. Comparison of the microbiological features of chronic and aggressive periodontitis. Periodontol. 2000, 53:70-88, 2010.

Bergström, J. Tobacco smoking and risk for periodontal disease. J. Clin. Periodontol., 30(2):107-13, 2003.

Botero, J. E.; González, A. M.; Mercado, R. A.; Olave, G. \& Contreras, A. Subgingival microbiota in peri-implant mucosa lesions and adjacent teeth in partially edentulous patients. J. Periodontol., 76(9):1490-5, 2005.

Buchmann, R.; Khoury, F.; Pingel, D. \& Lange, D. E. The microflora recovered from the outer-surfaces of the Frialit2 implanto-prosthetic connector. Clin. Oral Implants Res., 14(1):28-34, 2003.

De Boever, A. L. \& De Boever, J. A. Early colonization of non-submerged dental implants in patients with a history of advanced aggressive periodontitis. Clin. Oral Implants Res., 17(1):8-17, 2006.

Feloutzis, A.; Lang, N. P.; Tonetti, M. S.; Bürgin, W.; Brägger, U.; Buser, D.; Duff, G. W. \& Kornman, K. S. IL-1 gene polymorphism and smoking as risk factors for peri-implant bone loss in a well-maintained population. Clin. Oral Implants Res., 14(1):10-7, 2003.
Fürst, M. M.; Salvi, G. E.; Lang, N. P. \& Persson, G. R. Bacterial colonization immediately after installation on oral titanium implants. Clin. Oral Implants Res., 18(4):501-8, 2007.

Gerber, J.; Wenaweser, D.; Heitz-Mayfield, L.; Lang, N. P. \& Persson, G. R. Comparison of bacterial plaque samples from titanium implant and tooth surfaces by different methods. Clin. Oral Implants Res., 17(1):1-7, 2006.

Gruica, B.; Wang, H. Y.; Lang, N. P. \& Buser, D. Impact of IL1 genotype and smoking status on the prognosis of osseointegrated implants. Clin. Oral Implants Res., 15(4):393-400, 2004.

Hardt, C. R.; Gröndahl, K.; Lekholm, U. \& Wennström, J. L. Outcome of implant therapy in relation to experienced loss of periodontal bone support: a retrospective 5- year study. Clin. Oral Implants Res., 13(5):488-94, 2002.

Heuer, W.; Elter, C.; Demling, A.; Neumann, A.; Suerbaum, S.; Hannig, M.; Heidenblut, T.; Bach, F. W. \& StieschScholz, M. Analysis of early biofilm formation on oral implants in man. J. Oral Rehabil., 34(5):377-82, 2007.

Hultin, M.; Gustafsson, A.; Hallström, H.; Johansson, L. A.; Ekfeldt, A. \& Klinge, B. Microbiological findings and host response in patients with peri-implantitis. Clin. Oral Implants Res., 13(4):349-58, 2002.

Karoussis, I. K.; Salvi, G. E.; Heitz-Mayfield, L. J.; Brägger, U.; Hämmerle, C. H. \& Lang, N. P. Long-term implant prognosis in patients with and without a history of chronic 
periodontitis: a 10-year prospective cohort study of the ITI Dental Implant System. Clin. Oral Implants Res., 14(3):329-39, 2003.

Kocar, M.; Seme, K. \& Hren, N. I. Characterization of the normal bacterial flora in peri-implant sulci of partially and completely edentulous patients. Int. J. Oral Maxillofac. Implants, 25(4):690-8, 2010.

Leonhardt, A.; Renvert, S. \& Dahlén, G. Microbial findings at failing implants. Clin. Oral Implants Res., 10(5):339-45, 1999.

Leonhardt, A.; Gröndahl, K.; Bergström, C. \& Lekholm, U. Long-term follow-up of osseointegrated titanium implants using clinical, radiographic and microbiological parameters. Clin. Oral Implants Res., 13(2):127-32, 2002.

Leonhardt, A.; Dahlén, G. \& Renvert, S. Five-year clinical, microbiological, and radiological outcome following treatment of peri-implantitis in man. J. Periodontol., 74(10):1415-22, 2003.

Lindhe, J.; Meyle, J. \& Group D of European Workshop on Periodontology. Peri-implant diseases: Consensus Report of the Sixth European Workshop on Periodontology. J. Clin. Periodontol., 35(8 Suppl.):2825, 2008.

Mombelli, A. \& Décaillet, F. The characteristics of biofilms in peri-implant disease. J. Clin. Periodontol., 38 Suppl. 11: 203-13, 2011.

Norskov-Lauritsen, N. \& Kilian, M. Reclassification of Actinobacillus actinomycetemcomitans, Haemophilus aphrophilus, Haemophilus paraphrophilus and Haemophilus segnis as Aggregatibacter actinomycetemcomitans gen. nov., comb. nov., Aggregatibacter aphrophilus comb. nov. and Aggregatibacter segnis comb. nov., and emended description of Aggregatibacter aphrophilus to include $\mathrm{V}$ factor-dependent and $\mathrm{V}$ factor-independent isolates. Int. J. Syst. Evol. Microbiol., 56(Pt. 9):2135-46, 2006.

Nowzari, H.; Botero, J. E.; DeGiacomo, M.; Villacres, M. C. \& Rich, S. K. Microbiology and cytokine levels around healthy dental implants and teeth. Clin. Implant Dent. Relat. Res., 10(3):166-73, 2008.

Perea, E. J. Oral flora in the age of molecular biology. Med. Oral Patol. Oral Cir. Bucal, 9(Suppl.):6-10, 2004.

Quirynen, M.; Vogels, R.; Peeters, W.; van Steenberghe, D.; Naert, I. \& Haffajee, A. Dynamics of initial subgingival colonization of 'pristine' peri-implant pockets. Clin. Oral Implants Res., 17(1):25-37, 2006.

Renvert, S.; Roos-Jansåker, A. M.; Lindahl, C.; Renvert, H. \& Rutger Persson, G. Infection at titanium implants with or without a clinical diagnosis of inflammation. Clin. Oral Implants Res., 18(4):509-16, 2007.

Roos-Jansaker, A. M.; Renvert, S. \& Egelberg, J. Treatment of peri-implant infections: a literature review. J. Clin. Periodontol., 30(6):467-85, 2003.

Rutar A.; Lang, N. P.; Buser, D.: Bürgin, W. \& Mombelli, A. Retrospective assessment of clinical and microbiological factors affecting periimplant tissue conditions. Clin. Oral Implants Res., 12(3):189-95, 2001.

Salvi, G. E.; Fürst, M. M.; Lang, N. P. \& Persson, G. R. Oneyear bacterial colonization patterns of Staphylococcus aureus and other bacteria at implants and adjacent teeth. Clin. Oral Implants Res., 19(3):242-8, 2008.

Sumida, S.; Ishihara, K.; Kishi, M. \& Okuda, K. Transmission of periodontal disease-associated bacteria from teeth to osseointegrated implant regions. Int. J. Oral Maxillofac. Implants, 17(5):696-702, 2002.

Teughels, W.; Van Assche, N.; Sliepen, I. \& Quirynen, M. Effect of material characteristics and/or surface topography on biofilm development. Clin. Oral Implants Res., 17(Suppl. 2):68-81, 2006.

Van der Velden, U.; Varoufaki, A.; Hutter, J. W.; Xu, L.; Timmerman, M. F.; Van Winkelhoff, A. J. \& Loos, B. G. Effect of smoking and periodontal treatment on the subgingival microflora. J. Clin. Periodontol., 30(7):60310, 2003.

Van der Weijden, G. A.; van Bemmel, K. M. \& Renvert, S. Implant therapy in partially edentulous, periodontally compromised patients: a review. J. Clin. Periodontol., 32(5):506-11, 2005.

van Winkelhoff, A. J.; Goené, R. J.; Benschop, C. \& Folmer, T. Early colonization of dental implants by putative periodontal pathogens in partially edentulous patients. Clin. Oral Implants Res., 11(6):511-20, 2000.

Dirección para Correspondencia:

Dr. José Valdivia

Departamento de Odontología Restauradora

Universidad Autónoma de Chile

Santiago

CHILE

Email: doctorjosevaldiviaosorio@gmail.com

Recibido : 02-01-2014

Aceptado: 18-12-2014 